\title{
Importância da reavaliação primária seriada na condução do politraumatizado - relato de caso e revisão da literatura
}

\author{
The importance of the serial primary revaluation in the management \\ of polytrauma - case report and literature review
}

\author{
Bruna Queiroz Coelho ${ }^{1}$, Cristina Nery Carbajo ${ }^{1}$, Hüllie Hottgen Martins ${ }^{1}$, \\ Matheus Polly ${ }^{1}$, Rafael Furst ${ }^{2}$, Afonso César Polimanti², Mario Paulo Faro $\mathbf{J r}^{3}$
}

Coelho BQ, Carbajo CN, Martins HH, Polly M, Furst R, Polimanti AC, Faro Jr MP. Importância da reavaliação primária seriada na condução do politraumatizado - relato de caso e revisão da literatura / The importance of the serial primary revaluation in the management of polytrauma - case report and literature review. Rev Med (São Paulo). 2014 out.-dez.;93(4):159-64.

RESUMO: O estudo traz o relato de uma experiência de um paciente vítima de trauma carro versus bicicleta, que sofreu amputação traumática bilateral de membros inferiores. Para isso foram coletadas informações no banco de dados PubMed em 8 artigos, juntamente com informações da nova edição do livro ATLS ${ }^{\circledR}$, além de informações do paciente, do atendimento pré hospitalar e hopitalar, conduta e evolução. No caso exposto foi realizada correta avaliação primária e sua reavalição seriada, permitindo precoce identificação das lesões e conduta terapêutica, com boa evolução do paciente pós operatório. Afirma-se que a implementação do protocolo ATLS ${ }^{\circledR}$ é grande responsável pela diminuição da mortalidade do paciente politraumatizado. A reavaliação seriada se mostrou imprescindível para deteç̧ão do sangramento do paciente, estabilização e tratamento definitivo.

DESCRITORES: Cuidados de suporte avançado de vida no trauma; Amputação traumática; Reimplante; Choque hemorrágico; Vasos sanguíneos/lesões; Relatos de casos.

\begin{abstract}
The paper presentes the report of an accident patient of car $\mathrm{x}$ bicycle trauma who suffered traumatic amputation of the lower limbs. Therefore informations from eight articles of Pubmed database and new edition of ATLS book were collected, besides the preexisting informations from prehospital care, hospital care, conduct and evolution of the patient. The case described was carried out correct primary assessment and its serial reassessment, allowing early identification of lesions and therapeutic approach, with favorable postoperative patient. It is stated that the implementation of ATLS ${ }^{\circledR}$ protocol is largely responsible for the decrease in mortality of polytrauma patients. Serial reassessment proved to be indispensable for detection of patient bleeding, stabilization and final treatment.
\end{abstract}

KEYWORDS: Advanced trauma life support care; Amputation, traumatic; Replantation; Shock, hemorrhagic; Blood vessels/ lesions; Case reports.

Faculdade de Medicina do ABC. Categoria Prêmio ATLS.

1. Acadêmicos de Medicina da Faculdade de Medicina do ABC - FMABC, Santo André, SP.

2. Professores adjunto da Cirurgia Vascular da Faculdade de Medicina do ABC - FMABC, Santo André, SP.

3. Professor doutor regente da Disciplina Cirurgia Geral e de Urgência do Departamento de Cirurgia da Faculdade de Medicina do ABC - FMABC, Santo André, SP.

Endereço para correspondência: Bruna Queiroz Coelho. Rua Adolfo Laves, 275, Apart. 54 - Torre 2. Vila Valparaiso, Santo André, SP. CEP: 09060-390. E-mail: brunaqcoelho@hotmail.com 


\section{INTRODUÇÃO}

$\mathrm{O}$ trauma sempre foi um desafio internacional para os sistemas de saúde pública. De acordo com os últimos dados da Organização Mundial da Saúde (OMS) e do Centros de Controle e Prevenção de Doenças (CDC), morrem mais de 9 pessoas por minuto por trauma ou violência ${ }^{1}$. A Organização Mundial da Saúde (OMS) prevê para o ano de 2020 que os acidentes de trânsito serão a segunda maior causa externa de mortalidade no mundo ${ }^{2}$. O peso do trauma é ainda mais relevante no âmbito do custo global, representando $12 \%$ dos custos com doenças no mundo. Estima-se que os custos globais excedam 500 bilhões de dólares anualmente. Este valor seria ainda maior se considerasse que a população mais atingida são os jovens, ou seja, a camada mais produtiva na sociedade. No Brasil, ele é responsável por mais de 130 mil mortes por ano e por mais de 400 mil sequelas importantes, sendo suas vítimas principalmente jovens do sexo masculino ${ }^{2}$.

Adams Cowley, fundador do Instituto de Emergências Médicas de Maryland, criou o conceito da "hora de ouro". Ele verificou que os traumatizados que recebiam atendimento definitivo precoce tinham sobrevida muito maior do que aqueles que tinham seu tratamento definitivo postergado ${ }^{2}$. Para garantir a eficiência do atendimento precoce foi criado no fim da década de $70 \mathrm{o}$ protocolo ATLS $\AA$ (Advanced Trauma Life Support ${ }^{\circledR}$ ) que padronizou o seguimento do paciente politraumatizado, e seu advento permitiu uma análise mais objetiva dos casos, possibilitanto o rápido diagnóstico das alterações com alto risco de morte e seu tratamento ${ }^{1}$.

O programa do ATLS $\AA$ atualizado apoia-se em três conceitos: tratar primeiro a maior ameaça à vida, aplicar tratamento indicado mesmo na falta de diagnóstico e a não essencialidade da história detalhada para iniciar a avaliação do politraumatizado. O método é baseado na cronologia previsível de morte no trauma, por isso definiu-se a ordem de atenção seguindo o ABCDE. Os pacientes politraumatizados muitas vezes apresentam lesões exuberantes, como uma fratura exposta com sangramento ativo, capazes de desviar a atenção do médico da emergência que deixa de tratar adequadamente as vias aéreas. Entretanto, a obstrução destas é um quadro muito grave capaz de matar rapidamente o doente, evidenciando a importância de seguir a cronologia previsível de morte. Desse modo, a avaliação inicial deve ser rápida seguida de reavaliação periódica até a estabilização ou a necessidade da transferência do doente para um centro de trauma especializado.

A abordagem sistematizada, facilmente revista e aplicada consiste em preparação, triagem, avaliação primária $(\mathrm{ABCDE})$, reanimação, medidas auxiliares às primárias, necessidade de transferência do paciente, avaliação secundária e história, medidas auxiliares às secundárias, reavaliação e monitorização contínuas, e tratamento definitivo. Numa fase pré-hospitalar deve-se enfatizar a manutenção da via aérea, controle de hemorragia externa e do choque, imobilização do doente e transporte imediato ao hospital apropriado mais próximo. Em fase hospitalar deve estar disponível uma área de reanimação, equipamentos apropriados para abordagem de via aérea, soluções cristalóides aquecidas e equipamentos adequados de monitoração. $\mathrm{O}$ fato de existirem lesões musculoesqueléticas graves é grande preditor de que o paciente fora submetido a forças significativas durante o acidente, porém a presença deste trauma não indica que se deva reordenar o atendimento ATLS $®$. As lesões, no entanto, não devem ser ignoradas e para isso é preciso que seja realizado a reavaliação contínua do paciente para que as lesões sejam identificadas e tratadas no seu devido tempo. Em caso de lesão grave de extremidades, com risco potencial à vida, o tratamento da hemorragia arterial consiste em uma compressão direta do ferimento e reanimação com soluções necessárias. A interrupção sanguínea à uma extremidade deve ser prontamente reconhecida e as medidas terapêuticas devem ser tomadas emergencialmente. O eventual e singular uso de torniquete, quando apropriadamente aplicado, pode ser útil e salvar a vida do paciente, porém o tratamento definitivo sempre deve consistir no encaminhamento ao cirurgião. No caso de amputação traumática, uma forma grave de fratura exposta, há perda da extremidade, e trata-se de um evento traumático tanto físico quanto emocional para o paciente. A possibilidade de reimplante deve ser considerada, mediante análise das demais lesões do indivíduo, porém diante de lesões múltiplas, de necessidade de reanimação intensiva e de intervenção cirúrgica de urgência não é indicado o reimplante (habitualmente indicado em lesão isolada de extremidade, e de acordo com o escore de MESS - Mangled Extremity Severity Score). Além disso os pacientes politraumatizados, entre eles os amputados, são susceptíveis a perda de grande volume sanguíneo, com isso desencadeiam mecanismos de compensação fisiológicos para manter o débito cardíaco e evitar má perfusão tecidual. Para identificar o choque o ATLS® definiu o terceiro item chamado "circulation", no qual a atenção deve ser voltada para a frequência cardíaca e respiratória, a perfusão cutânea e a pressão de pulso. Os pacientes em choque iniciam o quadro com taquicardia e frio (vasoconstrição cutânea) e nessa altura o tratamento já deve ser estabelecido, de modo a controlar a hemorragia e repor o volume circulante. Portanto, é inquestionável que o atendimento à vítima do trauma deve-se iniciar mais precocemente possível e não sofrer descontinuidade. $\mathrm{O}$ atendimento deve ocorrer no local da ocorrência, sendo integrado aos cuidados durante o transporte das vítimas e ao tratamento definitivo que, em casos de politraumatizados, muitas vezes só é possível em ambiente hospitalar ${ }^{1}$. Este trabalho traz o relato de um caso que permite mostrar a importância do protocolo proposto 
Coelho BQ, et al. Importância da reavaliação primária seriada na condução do politraumatizado.

pelo ATLS $\AA$ além da indispensável reavaliação seriada do paciente, o que permitiu a boa evolução e sobrevivência do paciente no caso.

\section{MÈTODOS E PACIENTE}

Para o presente estudo será revisada a literatura na base de dados PubMed com os termos Cuidados de Suporte Avançado de Vida no Trauma; Amputação Traumática; Reimplante; Choque Hemorrágico e Vasos Sanguíneos/lesões, coleta de informações baseadas na $9^{\text {a }}$ edição do livro ATLS, além do relato de uma experiência de um paciente vítima de acidente que sofreu amputação traumática bilateral de membros inferiores. Para isso, foram submetidas informações do processo de atendimento pré hospitalar e hospitalar, de acordo com o protocolo ATLS $₫$, medidas de estabilização e tratamento definitivo.

Relato de um caso: Paciente do sexo masculino, 42 anos, vítima de colisão carro versus bicicleta. Na cena, foi encontrado caído 20 metros distante do local da colisão, demonstrando alta energia do acidente. No atendimento PHTLS: A) vias aéreas pérvias; B) ventilação espontânea; C) paciente hemodinamicamente instável com pressão arterial de $90 \times 70 \mathrm{mmHg}$ e pulso de 120 batimentos por minuto, identificado amputação traumática de membros inferiores bilateralmente com hemorragia ativa; D) pupilas normais e Glasgow 12; E) lesão nos membros inferiores, sem evidência de outros traumatismos. Como conduta foi mantida a via aérea pérvia com cânula de Guedel e máscara de oxigênio $100 \%$, foram feitos dois acessos venosos calibrosos com ringer lactato $2000 \mathrm{ml}$ e curativo compressivo em membros inferiores. Após essas medidas a pressão arterial passou para $110 \times 70 \mathrm{mmHg}$ e pulso para 100 batimentos por minuto, e foi feita analgesia com morfina. As pupilas continuaram normais, houve melhora do nível de consciência com Glasgow 15. Foi feita proteção com manta térmica por todo o corpo para evitar hipotermia. Foi encaminhado pelo resgate para o serviço terciário. $\mathrm{Na}$ chegada do paciente ao serviço, foi feita novamente a reavaliação primária seriada do paciente. A) vias aéreas pérvias. B) ventilação espontânea, taquipnéico (35 irpm), murmúrios vesiculares presentes bilateralmente sem ruídos, e sem abafamento de bulhas. Foi mantido cânula de Guedel e a máscara de oxigênio $100 \%$ com o objetivo de manter a saturação de $\mathrm{O}_{2}$ acima de $95 \%$; C) paciente pálido, com frialdade de extremidades, pulso de 140 batimentos por minuto, pressão arterial de $80 \times 40 \mathrm{mmHg}$ (mesmo após os $2000 \mathrm{ml}$ de ringer lactato do PHTLS), e sem hemorragia aparente no momento. Como paciente já apresentava dois acessos periféricos calibrosos e funcionantes, foi administrado mais $2000 \mathrm{ml}$ de Ringer Lactato aquecido, foi feita coleta de exames e solicitação de hemotransfusão com sangue tipo O liberado em caráter de emergência; D) Glasgow apresentado foi de 12 novamente e pupilas normais. $\mathrm{Na}$ exposição foram identificados curativos compressivos em membros inferiores completamente encharcados de sangue. Como se tratava de hemorragia intensa difícil de ser contida com curativo compressivo habitual, foi realizada imediatamente hemostasia com utilização de torniquetes elásticos (Faixa de Smarch), e aquecimento do paciente com manta térmica. Como medidas auxiliares ao atendimento primário foram realizados monitorização eletrocardiográfica, colocação de sondas nasogástrica e vesical, monitorização da oximetria de pulso, monitorização da frequência respiratória e gasometrial arterial. Foi feita nova reavaliação primária para avaliar resposta ao tratamento instituído. Paciente com vias aéreas pérvias e ventilação espontânea, com melhora da taquipnéia (frequência respiratória de 25 irpm e saturação de $\mathrm{O}_{2}$ de $94 \%$ ), melhora da palidez e frialdade, pressão arterial de $110 \times 70 \mathrm{mmHg}$, pulso de 100 batimentos por minuto, e diurese de $100 \mathrm{ml} /$ hora. Melhora do nível de consciência, com Glasgow de 15, e mantido manta térmica.

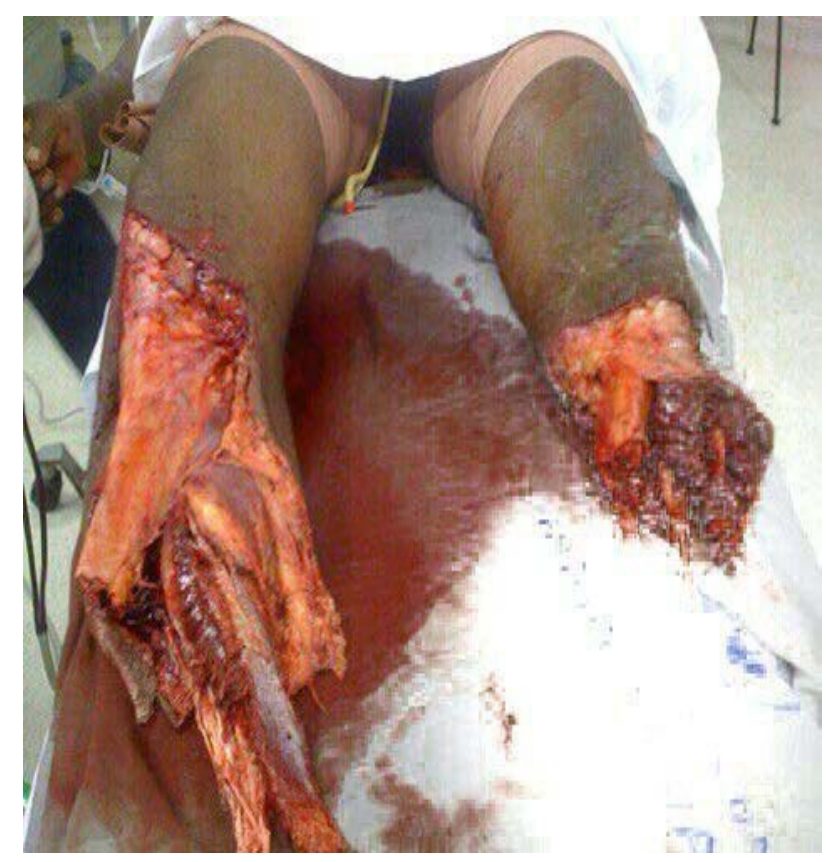

Figura 1. Amputação bilateral e garroteamento para contenção da hemorragia

Com a estabilização do paciente, prosseguiu-se com a avaliação secundária, coletando dados como histórico de alergias, medicamentos de uso habitual, passado médico, líquidos e alimentos ingeridos recentemente, ambiente e eventos relacionados ao trauma. No exame físico mais minucioso "da cabeça aos pés" não foi identificado nenhum outro tipo de lesão. Os exames de raio X de tórax, coluna cervical e tóraco-lombar, de bacia, e FAST (Focused Assessment Sonography in Trauma) de tórax e abdomen, todos sem alterações. Com a reavaliação global do paciente, este foi encaminhado ao centro cirúrgico, para tratamento definitivo com regularização dos cotos. 


\section{RESULTADOS}

Para avaliação das medidas tomadas com o paciente do caso apresentado, foram levantadas informações relacionadas ao assunto no banco de dados PubMed e na $9^{\text {a }}$ edição do ATLS $®$. No caso exposto o protocolo regido pelo ATLS foi seguido devidamente, com avaliação primária corretamente conduzida, identificação da hemorragia, estabilização do paciente e posterior resolução em centro cirúrgico para o tratamento definitivo, sendo este o reparo vascular e nervoso e regularização dos cotos com retalho músculo-cutâneo.

\section{DISCUSSÃO}

Estudos comparados das últimas décadas revelaram uma redução na taxa de mortalidade devido a hemorragia. A melhora na condução da hemorragia e implementação do ATLS ${ }^{\circledR}$ são grandes responsáveis pelas mudanças durante as últimas duas décadas. Van Olden et al. ${ }^{3}$ demonstrou que a introdução do programa ATLS $®$ reduziu significativamente a mortalidade durante os primeiros 60 minutos depois da admissão do paciente e identificou um número significativamente menor de pacientes com inadequado tratamento depois que o treinamento ATLS $®$ foi instituído. Meislin et al. ${ }^{4}$ reportou uma ditribuição bimodal da mortalidade com os picos acontecendo entre 60 minutos e 24-48h, respectivamente. Os autores assumiram que a distruibuição temporal da morte se tornou bimodal devido aos avanços tanto na conduta do trauma no pré hospitalar quanto no hospitalar.

Dano cerebral e hemorragia ainda são as mais importantes causas de morte pós trauma, apesar da mortalidade do politraumatizado por perda sanguínea ter sido reduzida. O diagnóstico precoce de hemorragia reduz a severidade do dano cerebral secundário. Essa melhora na sobrevivência por hemorragia aguda provavelmente é multifatorial, e a implementação do protocolo ATLS $®$ é um dos seus principais constituintes ${ }^{5}$. O reconhecimento do choque, reposição apropriada e a habilidade de encaminhar para o tratamento definitivo é vital na sequência da sobrevivência do trauma. Tradicionalmente, o choque é avaliado usando variações fisiológicas da pressão sanguínea sistólica, frequência cardíaca e pulso, como descrito pelos princípios do ATLS ${ }^{6}$. A reposição volêmica é iniciada depois de assegurar o acesso venoso, e consiste em solução de 1-2L de Ringer Lactato aquecido ou solução fisiológica. Os parâmetros que são importantes observar depois da reposição de fluidos incluem melhora da taquicardia, normalização da pressão arterial, melhora do nível de consciência, melhora do débito urinário e evidência global da melhora de perfusão de órgãos-alvo. Se o paciente demonstra apenas uma resposta mínima ou transitória à administração de fluidos, é sinal de sangramento permanente e outras estratégias de ressuscitação devem consistir de derivados do sangue, em vez de fluidos cristalóides ${ }^{7}$. No caso apresentado, paciente apresentava-se em choque classe IV, e necessitou além dos cristalóides, a hemotransfusão emergencial.

TABELA 1 - Perda estimada de sangue baseada na condição inicial do doente

\begin{tabular}{l|l|l|l|l}
\hline & Classe I & Classe II & Classe III & Classe IV \\
\hline Perda sanguínea (ml) & $<750$ & $750-1500$ & $1500-2000$ & $>2000$ \\
Frequência de pulso (bpm) & $<100$ & $100-120$ & $120-140$ & $>140$ \\
Pressão arterial & Normal & Normal & Diminuída & Diminuída \\
Pressão de pulso (mmHg) & Normal ou aumentada & Diminuída & $20-30$ & $30-40$ \\
Frequência respiratória & $14-20$ & $20-30$ & $5-15$ & Diminuída \\
Diurese (mL/h) & $>30$ & $\begin{array}{l}\text { Moderadamente } \\
\text { ansioso }\end{array}$ & Ansioso, confuso & Confuso, letárgico \\
Estado mental & Levemente ansioso & Cristalóide & Cristalóide e sangue & Cristalóide e sangue \\
\hline
\end{tabular}

Fonte: Adaptada da 9a edição do ATLS $®$, p.69.

Quando for tomada a decisão de fornecer produtos sanguíneos, sangue $\mathrm{O}$ positivo para os homens ou $\mathrm{O}$ negativo para as mulheres são prontamente disponíveis para infusão imediata até a prova de tipagem, em que o tipo específico é obtido do banco de sangue ${ }^{7}$. A perda de sangue é talvez a causa mais significante de morte potencialmente prevenível após injúria enquanto dano tecidual isquêmico leva à altas taxas de amputação em 
Coelho BQ, et al. Importância da reavaliação primária seriada na condução do politraumatizado.

população caracteristicamente jovem e ativa ${ }^{8}$. O grande desafio é saber quando investir na revascularização. Para isso, surgiram índices que buscaram estratificar a gravidade da lesão e predizer a necessidade de amputação. Helfet et al. ${ }^{9}$ e Sanders et al. ${ }^{10}$ (apud Guiraldo et al. ${ }^{11)}$ criaram um sistema de classificação das lesões por pontos, denominado índice MESS (Mangled Extremity Severity Score) ${ }^{11}$, que entre a literatura pesquisada, é o mais amplamente utilizado e possui poucas variáveis sendo de fácil aplicação. É um dos melhores preditores de não amputação (salvamento) de membro (MESS < 7). Em contrapartida índices $>7$ são correlacionados com $100 \%$ de amputação ${ }^{7}$.

QUADRO 1 - Critérios utilizados no Mangled Extremity Severity Score

\begin{tabular}{|l|l|c|}
\hline \multirow{5}{*}{ Lesões ósseas e de partes moles } & & Escore \\
\hline & Baixa energia: fraturas simples, lesões incisas & 1 \\
\cline { 2 - 3 } & Média energia: fraturas expostas, luxações & 2 \\
\cline { 2 - 3 } Isquemia dos membros* & Alta energia: esmagamentos, PAF de alta velocidade & 4 \\
\cline { 2 - 3 } & Presença de contaminação grosseira e avulsão de partes moles & 1 \\
\hline \multirow{5}{*}{ Choque } & Pulso diminuído ou ausente, mas com boa perfusão & 2 \\
\cline { 2 - 3 } & Ausência de pulsos, parestesias, diminuição do preenchimento capilar & 3 \\
\cline { 2 - 3 } & Ausência de pulso, frio e paralisia & \multirow{2}{*}{3} \\
\hline \multirow{5}{*}{ Idades } & PAS>90mmHg & 1 \\
\cline { 2 - 3 } & Hipotensão transitória & 2 \\
\cline { 2 - 3 } & Hipotensão persistente & 1 \\
\hline
\end{tabular}

*acima de 6h dobrar os pontos (Quadro 1 adaptado de Guiraldo et al. ${ }^{11}$ )

A necessidade de reconstrução da amputação de membros inferiores está aumentando, devido a traumas de alta energia em acidentes de estrada, como exposto no caso relatado. Comparado com reimplante de membros superiores, as indicações são mais seletas devido às frenquentes complicações nos procedimentos de salvamento dos membros inferiores ${ }^{12}$. Com base na literatura, as contra-indicações absolutas de reimplante são: tempo prolongado de isquemia, pacientes com alto risco cirúrgico e anestésico, procedimentos tecnicamente impossíveis devido às condições locais e trauma por avulsão ou esmagamento. A causa mais comum de contraindicação ainda é o dano extenso da parte amputada e/ou do coto proximal. Em média metade dos pacientes que sofreram amputação relacionada a trauma tem infecção da ferida ou necrose dela. Complicações no coto também são frequentes, além de neuromas sintomáticos e dor do membro fantasma ${ }^{13}$. Como resultado secundário da amputação, não apenas o membro é perdido, mas o paciente e familiares são frequentemente devastados e destruídos psicológico, físico, social e financeiramente ${ }^{14}$.

\section{CONCLUSÃO}

É evidente que a implementação do ATLS $®$ nas últimas décadas possibilitou um melhor e mais adequado tratamento frente ao paciente politraumatizado nas primeiras horas do acidente, levando à uma redução na taxa de mortalidade. Primeiramente o atendimento pré hospitalar foi muito bem conduzido no caso exposto, com identificação do mecanismo de trauma e sua intensidade, resolução momentânea do quadro e correta locomoção do paciente para o serviço terciário. A avaliação primária e sua revisão repetida a nível hospitalar foram de extrema 
importância para garantir a sobrevivência do paciente do caso relatado. A identificação precoce da hemorragia ativa em membros inferiores avulsionados e suas medidas terapêuticas, no caso o garroteamento, mesmo que não preconizado, foi responsável pela estabilização do paciente, permitindo o posterior tratamento definitivo. Apesar de se tratar de uma amputação traumática, que de acordo com

\section{REFERENCIAS}

1. American College of Surgeons. Advanced trauma life support ${ }^{\circledR}$ student course manual. Chicago: ACS Committee on Trauma; 2012.

2. Martins H, Damascero MC, Awada S. Pronto-socorro: medicina de emergência. 3a ed. São Paulo: Manole; 2012. p.605-6, p.620, 630-1.

3. Van Olden GDJ, Meeuwis JD, Bolhuis HW, et al. Clinical impact of advanced trauma life support. Am J Emerg Med. 2004;22:522-5. doi:10.1016/j.ajem.2004.08.013.

4. Meislin H, Criss EA, Judkins D, et al. Fatal trauma: the modal distribution of time to death is a function of patient demographics and regional resources. J Trauma. 1997;42:43340.

5. Pfeifer R, Tarkin I, Rocos B, Pape HC. Patterns of mortality and causes of death in polytrauma patients - Has anything changed? Injury. 2009;40(9):907-11. doi: 10.1016/j. injury.2009.05.006.

6. Lawton LD, Roncal S, Leonard E, Stack A, Dinh MM, Byrne CM, Petchell J. The utility of Advanced Trauma Life Support (ATLS) clinical shock grading in assessment of trauma. Emerg Med J. 2014;31(5):384-9. doi: 10.1136/ emermed-2012-201813.

7. Ray JM, Cestero R. Initial Management of the Trauma Patient. Atlas Oral Maxillofac Surg Clin North Am. 2013:21(1):1-7. doi: 10.1016/j.cxom.2012.12.005.

8. Perkins ZB, De'Ath HD, Aylwin C, Brohi K, Walsh M, Tai NR. Epidemiology and outcome of vascular trauma at a os critérios de MESS, por possuir score maior que 7 e portanto sem indicação de reimplante, o bem maior que é a vida do paciente fora salvo. Essa experiência é grandiosa em demonstrar a importância do seguimento rigoroso do ATLS ${ }^{\circledR}$ e sua incessante reavalição seriada, que no caso relatado garantiram o êxito no salvamento do paciente.

British Major Trauma Centre. Eur J Vasc Endovasc Surg. 2012;44(2):203-9. doi: 10.1016/j.ejvs.2012.05.013.

9. Helfet DL, Howey T, Sanders R, Johansen K. Limb salvage versus amputation. Preliminary results of the mangled extremity severity score. Clin Orthop Relat Res. 1990;(256):80-6 apud Guiraldo RP, Cabral C, Nora R, Gasques JA, Bozola RB. Trauma grave em membro inferior. Rev Bras Cir Plast. 2013;28(2):320-3. http://dx.doi. org/10.1590/S1983-51752013000200027.

10. Sanders R, Swiontkowski MF, Nunley JA 2nd, Spiegel PG. The management of fractures with soft-tissue disruptions. Instr Course Lect. 1994;43:559-70 apud Guiraldo RP, Cabral C, Nora R, Gasques JA, Bozola RB. Trauma grave em membro inferior. Rev Bras Cir Plast. 2013;28(2):320-3. http://dx.doi.org/10.1590/S1983-51752013000200027.

11. Guiraldo RP, Cabral C, Nora R, Gasques JA, Bozola RB. Trauma grave em membro inferior. Rev Bras Cir Plast. 2013;28(2):320-3. http://dx.doi.org/10.1590/S198351752013000200027 .

12. Battiston B, Tos P, Pontini I, Ferrero S. Lower limb replantations: indications and a new scoring system. Microsurgery. 2002;22(5):187-92. doi: 10.1002/micr.22505.

13. Tintle SM, Forsberg JA, Keeling JJ, Shawen SB, Potter BK. Lower extremity combat-related amputation. J Surg Orthop Adv. 2010;19(1):35-43

14. Shanmuganathan R. The utility of scores in the decision to salvage or amputation in severely injured limbs. Indian $\mathrm{J}$ Orthop. 2008;42(4):368-76. doi: 10.4103/0019-5413.43371. 